\title{
Ameliorative Effects of 3-methyladenine and Chloroquine in 3-nitropropionic-induced Huntington's Disease like Symptoms in Mice
}

\author{
Jasleen Kaur Virdi ${ }^{1}$, Ritu Kulshrestha ${ }^{2}$, Amteshwar Singh Jaggi ${ }^{1{ }^{*}}$ \\ ${ }^{1}$ Department of Pharmaceutical Sciences and Drug Research, Punjabi University Patiala, Patiala, Punjab, INDIA. \\ ${ }^{2}$ Department of Pathology, Vallabhbhai Patel Chest Institute, University of Delhi, New Delhi, INDIA.
}

\begin{abstract}
Aim: To study the effect of 3-methyladenine and chloroquine phosphate on 3-nitropropionic acid induced Huntington's disease-like symptoms in mice. Materials and Methods: 3-Nitropropionic acid was administered at the dose of $50 \mathrm{mg} / \mathrm{kg}$, i.p. twice daily for 5 days for inducing Huntington's disease like symptoms. 3-Methyladenine $(15$ and $30 \mathrm{mg} / \mathrm{kg}$, i.p.) and chloroquine phosphate $(25$ and $50 \mathrm{mg} / \mathrm{kg}$, i.p.) were administered $30 \mathrm{~min}$ before each 3-nitropropionic acid administration. The motor tests including, rota-rod, beam walking, lateral push test and open-field test, along with memory/cognitive test using object recognition test were performed on day 0,3 and 6 . The role of autophagy was assessed by measuring the levels of LC3II in the brain. Histopathological studies using $\mathrm{H} \& \mathrm{E}$, nissl staining; and immunohistochemistry of neuron specific enolase and caspase-3 were also performed. Results: Administration of 3-nitropropionic acid caused a decline in the motor functions and cognitive abilities of the animals. The histopathological studies also indicated neuronal injury and neuronal loss in the striatal region. Treatment with 3-methyladenine and chloroquine ameliorated motor and cognitive parameters induced by 3-nitropropionic acid along with prevention of neuronal loss. Moreover, these pharmacological agents ameliorated altered levels of LC3II with 3-methyladenine and chloroquine administration indicated involvement of autophagy. Conclusion: Treatment with 3-methyladenine and chloroquine may improve the symptoms related with Huntington's disease by preventing 3-nitropropionic acid-induced neurodegeneration possibly by inhibiting autophagy.
\end{abstract}

Key words: Huntington's disease, 3-nitropropionic acid, Autophagy, Chloroquine phosphate, 3-methyladenine.

\section{INTRODUCTION}

Huntington's disease (HD) is an autosomal-dominant inherited disorder in which neuro-degeneration occurs in the striatal region progressively over time, and is characterized by cognitive and motor impairment. ${ }^{1}$ The motor impairment includes imbalance, unexplained falls, hyperkinetic movements followed by hypokinesia, deterioration of gait and fine muscle movements causing difficulty in swallowing, which leads to decrease in body weight. ${ }^{2}$ Cognitive impairment displays as difficulty in learning new things, performing several things simultaneously and inability to retrieve memories ${ }^{3}$ The current treatment of HD patients involves tetrabenazine, amantadine clonazepam and olanzapine for motor improvements. ${ }^{4-6}$ However, the major limitation of the current treatment is that it provides only symptomatic relief to the patients ${ }^{6}$ and there is a need to identify new treatment strategies for the management of the disease. Autophagy is a highly regulated cellular process, which degrades faulty proteins and worn out organelles to recycle the resulting components. ${ }^{7}$ Under basal conditions, cellular autophagy is generally low and
Submission Date: 22-04-2021; Revision Date: 02-06-2021; Accepted Date: 10-09-2021

DOI: 10.5530/ijper.55.4.204 Correspondence: Dr. Amteshwar Singh Jaggi Department of Pharmaceutical Sciences and Drug Research, Punjabi University Patiala, Patiala-147002, Punjab, INDIA.

Phone: +91-9501016036, Email - amteshwarjaggi@ yahoo.co.in

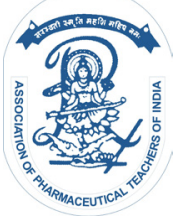

www.ijper.org 
serves housekeeping function to maintain normal homoeostasis. ${ }^{8}$ Apart from the physiological role of autophagy, studies have also shown its involvement in the pathogenesis of different diseases. Although there have been studies showing the beneficial effects of autophagy in neurodegenerative diseases, ${ }^{9,10}$ yet excessive activation of autophagy has been documented to produce deleterious effects due to induction of apoptosis and degradation of essential proteins of the cell. ${ }^{11-13} \mathrm{~A}$ distinct role of autophagy has been identified to be a maladaptive response to hemodynamic stress on myocytes which accentuates pathological remodeling. ${ }^{14}$ Excessive activation of autophagy is shown to induce neuronal death in ischemia-reperfusion induced injury in the PC12 cells and hippocampus region of rats. ${ }^{15,16}$ More studies have shown that activation of autophagy may induce neurodegeneration ${ }^{13}$ and neuronal cell death. ${ }^{17}$

Moreover, it has been shown that 3-nitropropionic acid, a well-documented chemical inducer of Huntington's disease, induces striatal neuronal degeneration by promoting mitochondrial damage and activating autophagy. Indeed, 3-nitropropionic acid is a mitochondrial toxin, which deprives the neurons of energy and induces autophagy to produce neurochemical and histological changes corresponding to that in the Huntington's disease. ${ }^{18-20}$ Therefore, it is possible that autophagic inhibition may overcome the behavioral, cognitive decline and neuronal injury induced by 3-nitropropionic acid. Therefore, the present study aims to investigate the ameliorative effects of autophagic inhibitors, 3-methyladenine and chloroquine on 3-nitropropionic-induced Huntington's disease like symptoms in mice.

\section{MATERIALS AND METHODS}

\section{Experimental animals}

Swiss albino mice (CRI, Kasauli, India) of either sex weighing 20-25 g, maintained at standard laboratory diet (Ashirwad Feeds Ltd., Kharar, India) with free access to tap water, were employed in the study. Mice were accommodated in the animal house of the department and were exposed to normal light and dark cycle. The experimental studies were blinded for allocation of animals, treatment and data analysis. Institutional Animal Ethics Committee (107/GO/ReBi/S/99/ CPCSEA/2018-05) duly approved the experimental protocol and the animals were taken care of as per the guidelines by Committee for the Purpose of Control and Supervision of Experiments on Animals (CPCSEA),
Ministry of Environment and Forest, Government of India (Reg. No. 107/1999/CPCSEA).

\section{Drugs and chemicals}

3-Nitropropionic acid (Sigma Aldrich, USA), 3-methyladenine (Sigma Aldrich, USA) and chloroquine phosphate (Ipca Laboratories Ltd. Mumbai) were dissolved in normal saline. All the chemicals and analytical grade reagents were employed in this study. The doses of 3-nitropropionic acid, 21-23 3-methyladenine ${ }^{24,25}$ and chloroquine phosphate ${ }^{26,27}$ were selected on the basis of previously published studies.

\section{Acclimatization of the animals}

The experimental animals were acclimatized to the test apparatus and the laboratory 3 days prior to beginning the experimental protocol.

\section{Induction of Huntington's disease}

The animals were administered 3-nitropropionic acid $(50 \mathrm{mg} / \mathrm{kg}$, i.p.) twice daily for 5 days to induce the symptoms pertaining to Huntington's disease in mice. $22,21,28$

\section{Behavioral examination}

The base line readings for the behavioral tests and body weight were recorded on day 0 i.e., before the first 3-nitropropionic acid injection on day 1 . The behavioral test readings and body weight were recorded on the $3^{\text {rd }}$ day before injection and on the $6^{\text {th }}$ day.

\section{Grip strength (Rota rod test)}

Rota-rod test was employed to assess the grip strength of the animals. The time for which the mice stay on the rotating rod of the apparatus is considered as the marker of their grip strength. The fall off latency of the animals was used as an index of grip strength. The cut off period was set at 300 seconds. $^{29}$

\section{Motor coordination and balance (Beam walking test)}

This test was used for assessing the motor coordination and balance in the animals. The animals were made to walk on an elevated narrow path. The time taken to cross the path and the number of foot slips were noted. The cut off time for the animals to traverse across the beam was 60 seconds. ${ }^{30}$

\section{Motor activity (Lateral push test)}

The motor activity and grip strength of the animals was assessed using lateral push test. The response of the animals to the push stimuli applied laterally on either of the shoulders was assessed..$^{31}$ For the purpose of having a firm grip, the animals were placed on a rough platform. The mice were scored from 0-3 depending on 
the resistance shown by the mice, with 0 representing no injury and 3 representing the maximal injury. ${ }^{32}$

\section{Mobility behavior in a modified Open field test}

The open field test was employed for the assessment of animal mobility over a period of five minutes. Immobility is one of the characteristic features of Huntington's disease. The immobility period of the animals was recorded with the cut off period of five minutes. ${ }^{33}$

\section{Memory evaluation (Object recognition test)}

Object recognition is one of the commonly employed memory test. ${ }^{34,35}$ The object recognition test was performed in the same arena as for open field test. The animals were subjected to three sessions i.e., habituation session on day 4, familiarization session $24 \mathrm{hr}$ after habituation session i.e., on day 5 and test session was performed $24 \mathrm{hr}$ after the familiarization session on day 6. During the habituation session, the animals were left in the arena to explore it for a duration of $5 \mathrm{~min}$. During the familiarization session, two objects were introduced in the arena and the animals were allowed to explore them for a period of $5 \mathrm{~min}$. After $24 \mathrm{hr}$, during the test session one of the objects in the arena was substituted with a new object. The animals were again allowed to explore the objects for $5 \mathrm{~min}$. Discrimination index was determined by noting the difference between the time spent by the animal in exploring the new and the familiar object. ${ }^{34}$

$$
\begin{aligned}
\text { Discrimination index }= & \left(\mathrm{T}_{\text {Novel }}-\mathrm{T}_{\text {Familiar }}\right) / \\
& \left(\mathrm{T}_{\text {Novel }}+\mathrm{T}_{\text {Familiar }}\right) \times 100 \%
\end{aligned}
$$

\section{Isolation of brain}

On the $6^{\text {th }}$ day after completion of the behavioral tests, the animals were sacrificed by cervical dislocation and the brains were carefully removed. The two hemispheres of the brains were separated and one-half of each brain was used for histopathological studies (discussed below) and the other half was used for carrying out the biochemical estimation (discussed below). The halves of the brains used for the histopathological studies were preserved in $10 \%$ buffered formalin solution at $4^{\circ} \mathrm{C}$. The other halves of the brains used for biochemical estimation were homogenized in freshly prepared phosphate buffer $(\mathrm{pH}=7.4)$ and centrifuged at $654.03 \mathrm{~g}(3000 \mathrm{rpm})$ for $15 \mathrm{~min}$ to obtain the supernatant. The biochemical estimation was performed using the supernatant.

\section{Biochemical estimation}

\section{Estimation of LC3II levels}

The LC3II levels in the brain homogenate were estimated using ELISA for assessing the extent of autophagy in the brain. ${ }^{36}$ The instructions provided by the ELISA kit manufacturer were used for carrying out the assay.

\section{Histopathological studies}

\section{Niss/ staining (cresyl violet staining)}

Extent of atrophy in the striatal regions of the brains was estimated by staining them with cresyl violet dye. ${ }^{37,38}$

\section{Hematoxylin and eosin staining (H\&E)}

Hematoxylin and eosin staining gives an estimate of the extent of neuronal loss in the striatal area of the brain which is most prone to damage in HD. ${ }^{38,39}$

\section{Immunohistochemistry}

\section{Neuron specific enolase (NSE)}

NSE, a glycolytin enzyme present in the neuronal tissue is an efficient marker of neuronal injury and neuronal cell death. ${ }^{40}$ The samples of the brain were sent to 'Vallabhbhai Patel Chest Institute, New Delhi'.

\section{Caspase 3}

Caspases are one of the important proteolytic enzymes which play a key role in apoptosis. ${ }^{41}$ The extent of apoptosis in the brain samples was assessed by estimating the activity of caspase $3 .^{41}$

\section{Experimental protocol}

Forty-two Swiss mice were equally distributed amongst seven groups for the present study.

\section{Group I: Normal Control}

The animals in this group were not treated with any of the drugs used in this study. Mice were evaluated for behavioral parameters and their body weight was recorded on day 0 , day 3 and 6 . The object recognition test was carried on day 4, 5 and 6 as described above. On $6^{\text {th }}$ day the animals were sacrificed and the brains were removed for biochemical and histopathology testing.

\section{Group II: HD control}

3-Nitropropionic acid $(50 \mathrm{mg} / \mathrm{kg}$, i.p.) was administered to the animals twice daily for 5 days. The behavioral, biochemical and histopathology testing were performed as mentioned in group I.

\section{Group III: Choloroquine phosphate treatment $(25 \mathrm{mg} / \mathrm{kg}$, i.p.) in $H D$}

The animals were treated with chloroquine phosphate $(25 \mathrm{mg} / \mathrm{kg}$, i.p. $)$ daily $30 \mathrm{~min}$ before each 3-nitropropionic acid administration. The same protocol was followed for testing of behavioral parameters, histopathology and biochemical testing as mentioned in group I. 


\section{Group IV: Chloroquine phosphate treatment (50 $\mathrm{mg} / \mathrm{kg}$, i.p.) in $\mathrm{HD}$}

Over the period of 5 days, the animals were treated with chloroquine phosphate $(50 \mathrm{mg} / \mathrm{kg}$, i.p. $)$ daily $30 \mathrm{~min}$ before each 3-nitropropionic acid administration. The same protocol was followed for testing of behavioral parameters, histopathology and biochemical testing as mentioned in group I.

\section{Group V: 3-methyl adenine treatment (15 mg/kg, i.p.) in $H D$}

Over the period of 5 days, the animals were treated with 3-methyl adenine $(15 \mathrm{mg} / \mathrm{kg}$, i.p.) daily $30 \mathrm{~min}$ before each 3-nitropropionic acid administration. The same protocol was followed for testing of behavioral parameters, histopathology and biochemical testing as mentioned in group I.

\section{Group VI: 3-methyl adenine treatment (30 mg/kg, i.p.)} in $H D$

Over the period of 5 days, the animals were treated with 3-methyl adenine $(30 \mathrm{mg} / \mathrm{kg}$, i.p.) daily $30 \mathrm{~min}$ before each 3-nitropropionic acid administration. The same protocol was followed for testing of behavioral parameters, histopathology and biochemical testing as mentioned in group I.

\section{Group VII: Chloroquine phosphate (50 mg/kg, i.p.) per se}

The animals in this group were treated with chloroquine phosphate $(50 \mathrm{mg} / \mathrm{kg}$, i.p.) twice daily for five days. The same protocol was followed for testing of behavioral parameters, histopathology and biochemical testing as mentioned in group I.

\section{Group VIII: 3-methyladenine (30 mg/kg, i.p.) per se}

The animals in this group were treated with 3-methyladenine $(30 \mathrm{mg} / \mathrm{kg}$, i.p.) twice daily for five days. The same protocol was followed for testing of behavioral parameters, histopathology and biochemical testing as mentioned in group I.

\section{Statistical analysis}

The results obtained were expressed as mean \pm S.D. The data obtained from behavioral tests was analyzed statistically by two-way analysis of variance (ANOVA) followed by Tukey's multiple comparison test. Oneway ANOVA was used for statistical analysis of data obtained from biochemical tests followed by Tukey's multiple comparison test. Statistical analysis was performed using Graph Pad Prism version 8.0 software. The $p$ value $<0.05$ was considered to be statistically significant.

\section{RESULTS}

Effect of pharmacological interventions on the motor functions in 3-nitropropionic acid administered mice

Administration of 3-nitropropionic acid $(50 \mathrm{mg} / \mathrm{kg}$, i.p.) twice daily for five days significantly impaired the motor functions in mice assessed on $3^{\text {rd }}$ and $6^{\text {th }}$ day of 3-nitropropionic acid administration in comparison to day 0. 3-Nitropropionic acid led to significant impairment in the grip strength of the animals assessed using rota rod test (Figure 2a); increase in the number of foot slips (Figure 1a) and time taken to cross the beam (Figure 1b) assessed using beam walking test; increase in the immobility time in the open field test (Figure 3a); and decrease in the resistance to the lateral push test (Figure $2 \mathrm{~b}$ ). The motor impairment was more significant on $6^{\text {th }}$ day in comparison to the $3^{\text {rd }}$ day of 3-nitropropionic acid administration.

Treatment with 3-MA (15 and $30 \mathrm{mg} / \mathrm{kg}$, i.p.) for five days before each 3-nitropropionic acid injection significantly attenuated 3-nitropropionic acid induced impairment of motor functions in a dose-dependent manner assessed on $3^{\text {rd }}$ and $6^{\text {th }}$ day and chloroquine phosphate $(50 \mathrm{mg} / \mathrm{kg}$, i.p.) treatment also improved the motor functions in 3-nitropropionic acid administered

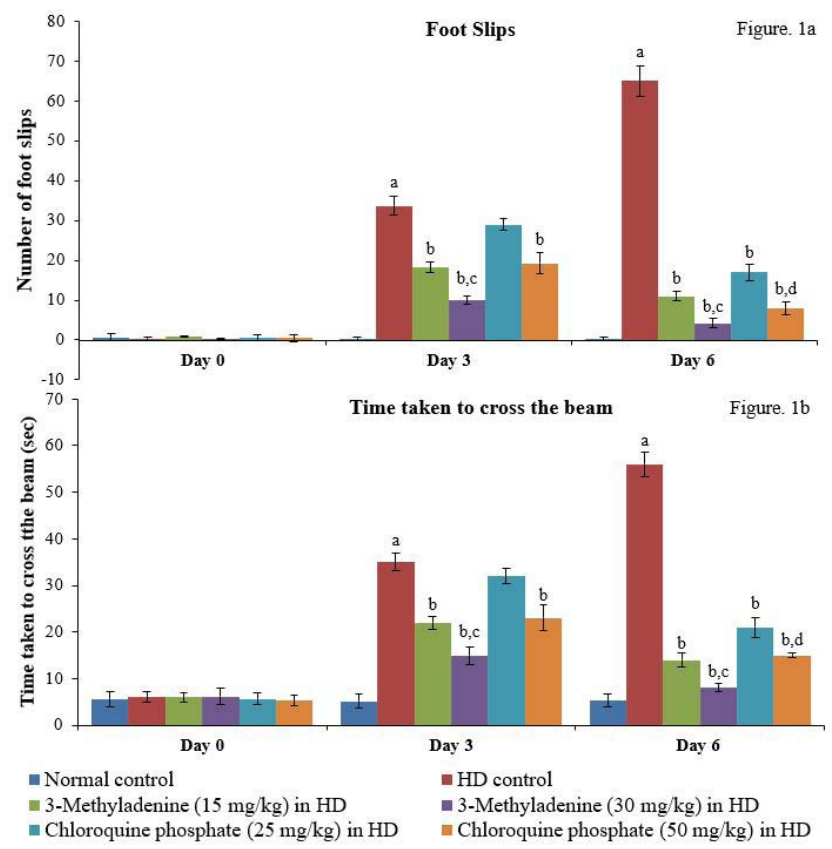

Figure 1: Effect of the pharmacological interventions on the motor coordination and balance (Figure 1a). Time taken to cross the beam (Figure $1 \mathrm{~b}$ ). $a=P<0.0001$ as compared to normal control; $\mathrm{b}=P<0.0001$ as compared to HD control; $\mathrm{C}=P<0.05$ as compared to 3-methyladenine $(15 \mathrm{mg} /$ $\mathrm{kg}) ; \mathrm{d}=P<0.0001$ as compared to chloroquine phosphate $(25 \mathrm{mg} / \mathrm{kg})$. 1a) $[F(2,90)=783.1]$ for the time factor and $[F(5,90)=495.2]$ for treatment factor; $1 \mathrm{~b})[F(2,90)=749.8]$ for the time factor and $[F(5,90)=426.3]$ for treatment factor. 

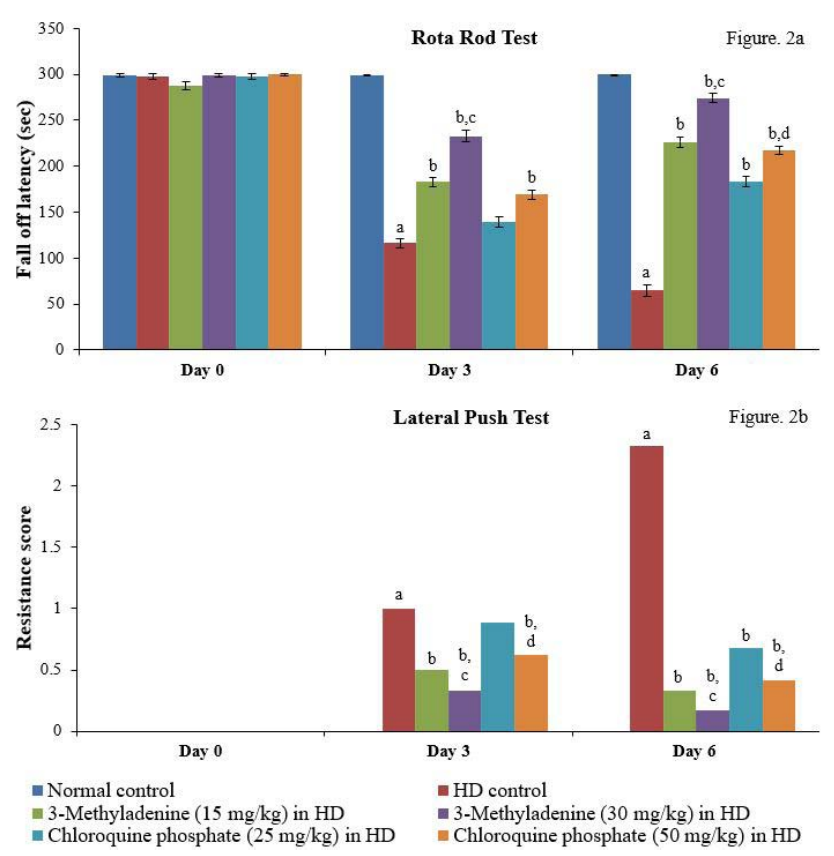

Figure 2: Effect of the pharmacological interventions on the grip strength of the animals using rotarod test (Figure 2a) and motor activity of the animals in terms of resistance to the lateral push (Figure 2b) $a=P<0.0001$ compared to normal control; $\mathrm{b}=P<0.0001$ compared to HD control; $\mathrm{c}=P<0.0001$ compared to 3-methyladenine (15 mg/kg); $\mathrm{d}=P<0.0001$ compared to chloroquine phosphate $(25 \mathrm{mg} / \mathrm{kg})$. 2a) $[F(2,90)=7334]$ for the time factor and $[F(5,90)=2618]$ for the treatment factor; $2 b)[F(2,90)=29285]$ for the time factor and $[F(5,90)=17499]$ for the treatment factor.

animals on $3^{\text {rd }}$ and $6^{\text {th }}$ day, while the significant effects of chloroquine phosphate $(25 \mathrm{mg} / \mathrm{kg}$, i.p. $)$ were observed only on $6^{\text {th }}$ day of its administration. Moreover, the beneficial effects of 3-MA were relatively more pronounced in 3-nitropropionic acid administered mice comparison to CQ (Figures 1a, 1b, 2a, 2b, 3a).

\section{Effect of pharmacological interventions on the memory in 3-nitropropionic acid administered mice}

The ability to distinguish between the novel and familiar object as an index of memory was significantly decreased in 3-nitropropionic acid administered mice in terms of decrease in discrimination index assessed on the $6^{\text {th }}$ day in the object recognition test. In other words, the discrimination index was significantly decreased in 3-nitropropionic acid injected mice in comparison to normal control animals (Figure 3b). Treatment with 3-MA (15 and $30 \mathrm{mg} / \mathrm{kg}$, i.p.) before each 3-nitropropionic acid administration for five days showed a dose-dependent improvement in the cognition of the animals in terms of increase in the discrimination index assessed on $6^{\text {th }}$ day. Chloroquine phosphate (25 and $50 \mathrm{mg} / \mathrm{kg}$, i.p.) also elicited an
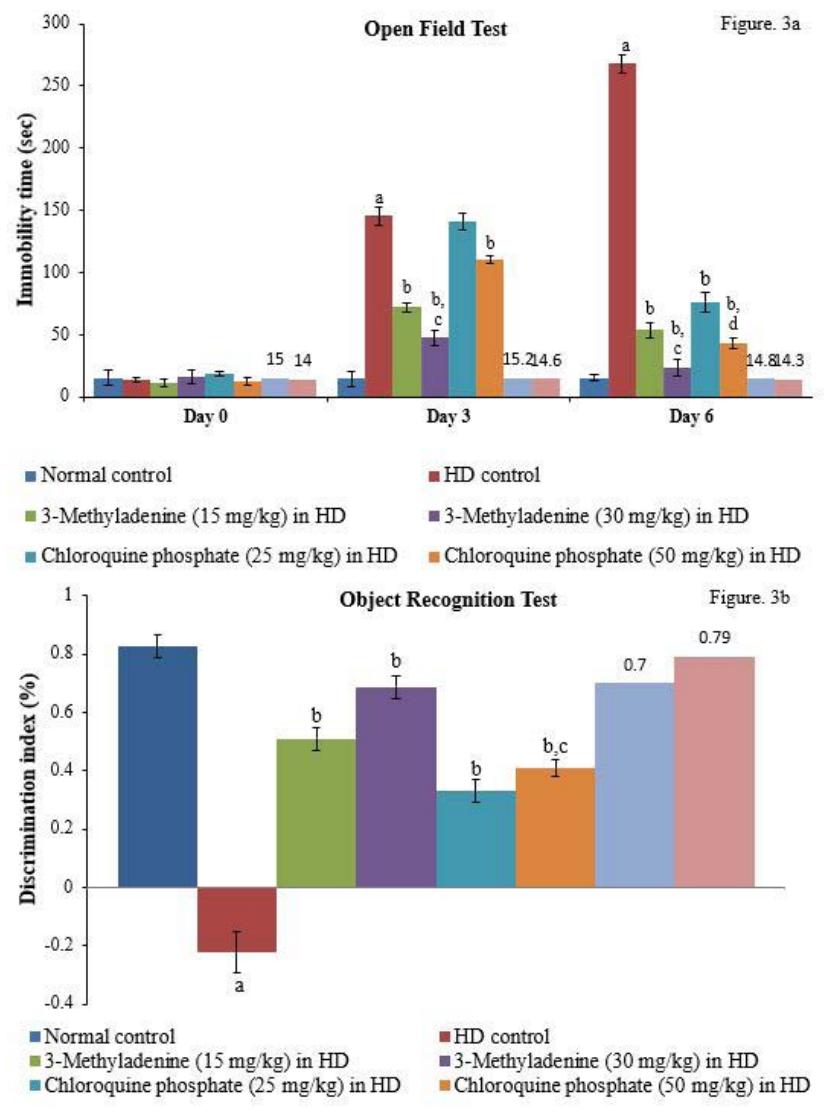

Figure 3: Effect of pharmacological interventions on motor functions of animals in terms of immobility time using openfield test (Figure 3a) b) cognition of the animals using object recognition test. Values were expressed as mean \pm S.D. $(n=6)$ and data were analyzed by Two-way ANOVA (Figure 3a) and One-way ANOVA (Figure $3 b$ ), $a=P<0.0001$ compared to normal control; $\mathrm{b}=P<0.0001$ compared to $\mathrm{HD}$ control; $\mathrm{c}=P<0.0001 \mathrm{com}$ pared to 3-MA (15 mg/kg); $\mathrm{d}=P<0.0001$ compared to $C Q(25 \mathrm{mg} /$ kg). 3a) $[F(2,90)=6599]$ for the time factor and $[F(5,90)=4177]$ for the treatment factor; $3 b)[F(5,30)=57]$ for the treatment factor.

improvement in the cognitive ability of HD animals in a dose-dependent fashion. However, the effect was more significant with 3-MA treatment in comparison with chloroquine phosphate treatment (Figure $3 \mathrm{~b}$ ).

\section{Effect of pharmacological interventions on the levels of LC3II, a marker of autophagy}

Administration of 3-nitropropionic acid $(50 \mathrm{mg} / \mathrm{kg}$, i.p.) twice daily for five days significantly increased the levels of LC3II in the brain samples of mice in comparison to the normal control. Treatment with 3-methyladenine (15 and $30 \mathrm{mg} / \mathrm{kg}$, i.p.) elicited a significant dose-dependent decline in LC3II levels. However, chloroquine phosphate $(25$ and $50 \mathrm{mg} / \mathrm{kg}$, i.p.) treatment significantly increased the levels of LC3II (Figure 4). 


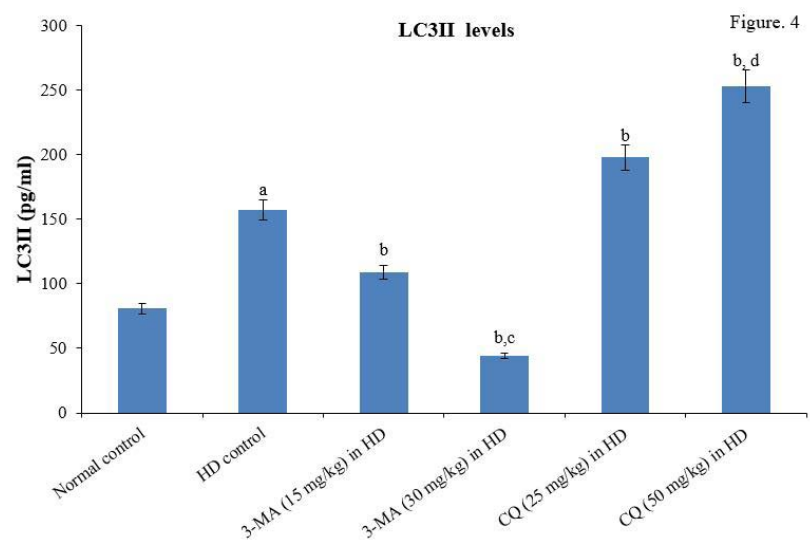

Figure 4: Effect of pharmacological modulators on the levels of LC3II Values were expressed as mean \pm S.D. $(n=6)$ and data were statistically analyzed by One-way ANOVA followed by post-hoc analysis using Tukey's Multiple Comparison test, $a=P<0.0001$ as compared to normal control; $b=P<0.0001$ as compared to HD control; $\mathrm{C}=P<0.0001$ as compared to 3-methyladenine $(15 \mathrm{mg} / \mathrm{kg}) ; \mathrm{d}=P<0.0001$ as compared to chloroquine phosphate $(25 \mathrm{mg} / \mathrm{kg})$. $[F(5,30)=4924]$ for the treatment factor.

\section{Effect of pharmacological interventions on the body} weight in 3-nitropropionic acid administered mice

There was an increase in the weight gain in the normal mice over a period of six days. However, there was a significant decrease in the body weight in 3-nitropropionic acid administered mice as observed on $3^{\text {rd }}$ and $6^{\text {th }}$ day. Treatment with 3 -MA (15 and $30 \mathrm{mg} / \mathrm{kg}$, i.p.) significantly improved the body weight in 3-nitropropionic acid administered mice, whereas, treatment with chloroquine phosphate $(25$ and $50 \mathrm{mg} / \mathrm{kg}$, i.p.) prevented 3-nitropropionic acid induced loss in body weight on $3^{\text {rd }}$ and $6^{\text {th }}$ day.

\section{Effect of pharmacological interventions on neuronal loss and brain atrophy in brain samples of 3-nitropropionic acid administered mice}

$\mathrm{H}$ and $\mathrm{E}$ staining also showed a significant increase in the percentage loss of neurons in the brain samples of 3-nitropropionic acid administered mice. Treatment with 3-MA (15 and $30 \mathrm{mg} / \mathrm{kg}$, i.p.) and chloroquine phosphate $(25$ and $50 \mathrm{mg} / \mathrm{kg}$, i.p.) significantly increased the number of positively stained neurons. However, 3-MA treatment prevented 3-nitropropionic acid induced neuronal loss much more efficiently in comparison with chloroquine phosphate treatment (Figure 5).

Nissl staining performed on the brain samples of the mice administered with 3-nitropropionic acid showed a decrease in the amount of Nissl substance and number of neurons, particularly in the striatal region. However, in the brain sections of animals treated with 3-MA (15 and $30 \mathrm{mg} / \mathrm{kg}$, i.p.) showed an improvement

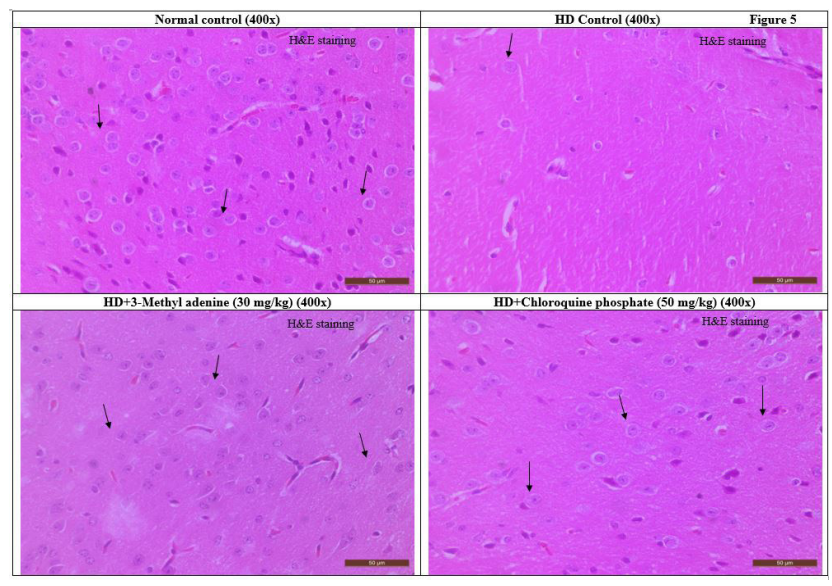

Figure 5: Effect of pharmacological modulators on the extent of neuronal loss assessed using Hematoxylin and eosin staining (400x). Arrow: Normal neurons.

in the amount of Nissl substance and increase in the number of viable neurons. Treatment with chloroquine phosphate ( 25 and $50 \mathrm{mg} / \mathrm{kg}$, i.p.) also prevented the loss of Nissl bodies and neuronal cells in 3-nitropropionic acid administered mice (Figure 6).

Effect of the pharmacological interventions on NSE and caspase 3 activity in the brain samples of 3-nitropropionic acid administered mice

Immunohistochemistry of the brain samples of 3-nitropropionic acid administered mice showed a marked increase in the NSE expression in comparison to normal control, in which only occasional neurons showed NSE expression. Treatment with 3-MA (15 and $30 \mathrm{mg} / \mathrm{kg}$, i.p.) and chloroquine phosphate $(50 \mathrm{mg} / \mathrm{kg}$, i.p.) before each 3-nitropropionic acid administration for five days significantly decreased the NSE expression in the brain samples. The effects of 3-MA were more pronounced in comparison to chloroquine phosphate treatment (Figure 7).

Similarly, brain samples of 3-nitropropionic acid treated mice showed a significantly increased number of neurons which stained positive for caspase 3 in comparison to normal control group in which only occasional neurons stained positive for caspase 3 . Five days treatment with 3-MA (15 and $30 \mathrm{mg} / \mathrm{kg}$, i.p.) and chloroquine phosphate $(50 \mathrm{mg} / \mathrm{kg}$, i.p.) produced a significant decline in the caspase 3 activity as the brains samples of these groups showed less number of neurons positively stained for caspase 3 (Figure 8).

\section{Effect of pharmacological interventions in the normal animals and vehicle administration in 3-nitropropionic acid induced $H D$ animals}

Per se administration of chloroquine phosphate (50 $\mathrm{mg} / \mathrm{kg}$, i.p.) and 3-methyladenine $(30 \mathrm{mg} / \mathrm{kg}$, i.p.) did 

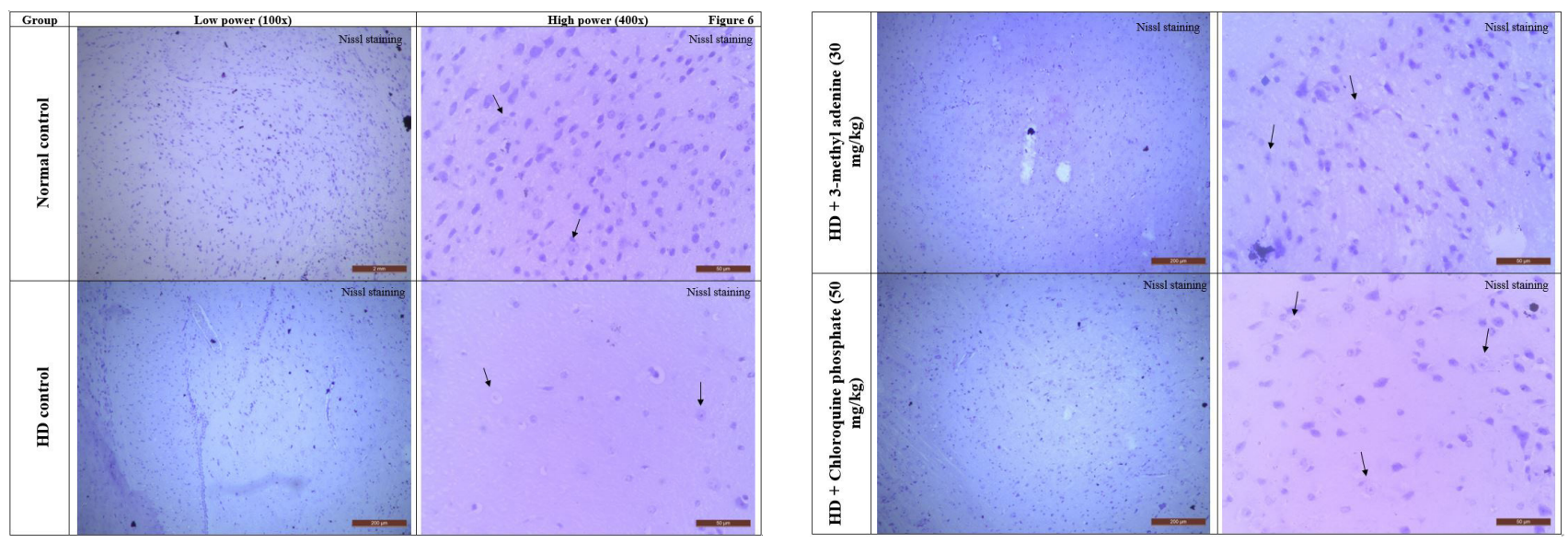

Figure 6: Effect of pharmacological modulators on the extent of neuronal atrophy assessed using Nissl staining (100x and 400x). Arrow: Normal neurons.
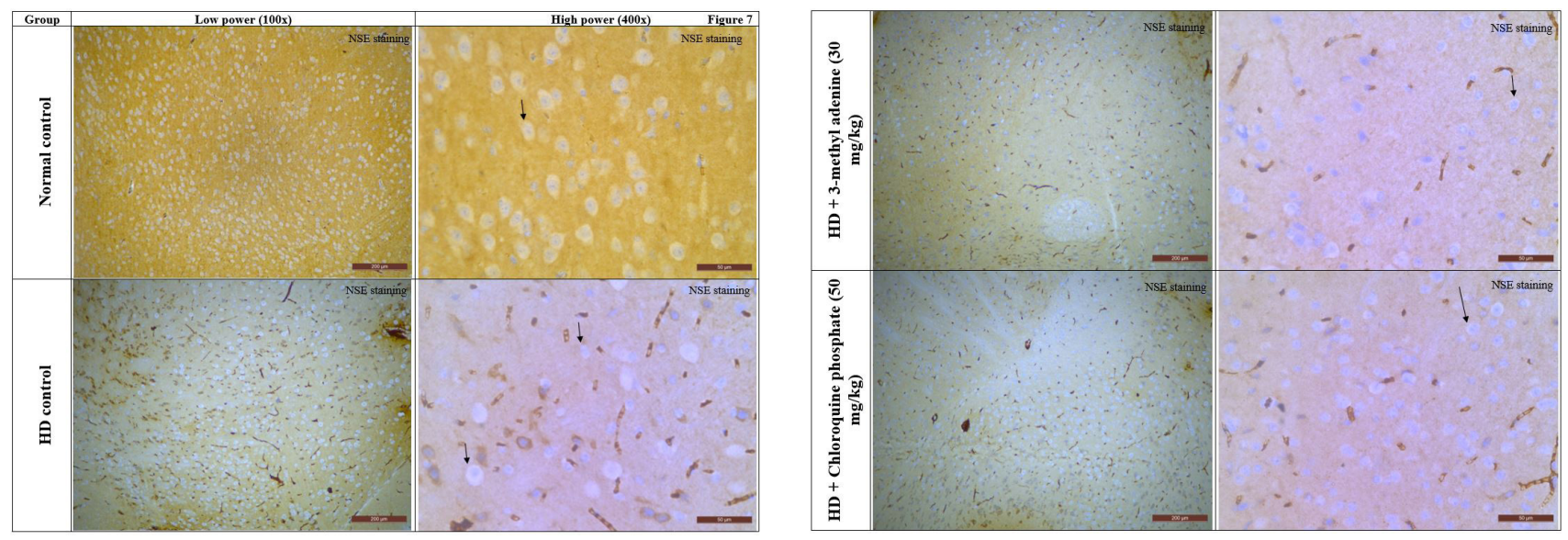

Figure 7: Effect of pharmacological modulators on neuronal inflammation assessed using NSE immunohistochemistry (100x and 400x). Arrow: Normal neurons.
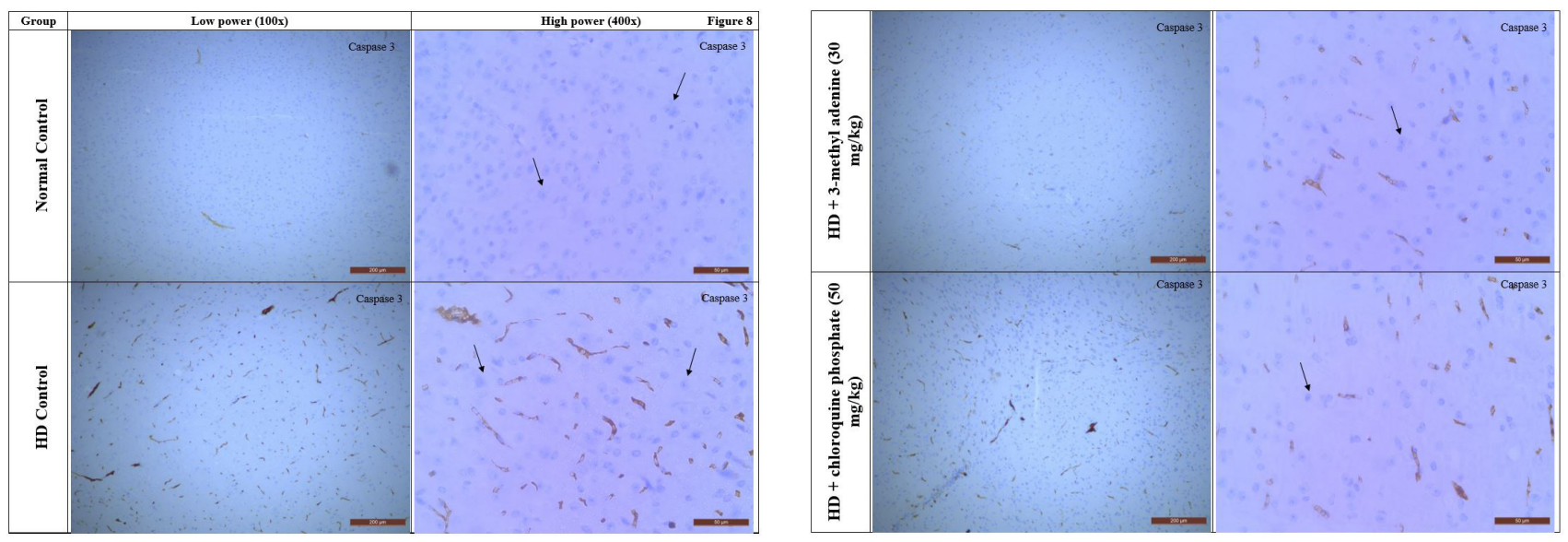

Figure 8: Effect of pharmacological modulators on the extent of apoptosis assessed by caspase 3 activity using immunohistochemistry (100x and 400x). Arrow: Normal neurons. 
not significantly affect the body weight, behavioral parameters, biochemical and histopathological parameters of the normal animals. Moreover, treatment with vehicle in 3-nitropropionic acid administered mice did not significantly affect the body weight, behavior of the animals, the biochemical and the histopathological parameters. There were no drop-outs or animal mortalities observed in any experimental group of our study.

\section{DISCUSSION}

In this study, 3-nitropropionic acid administration at the dose of $50 \mathrm{mg} / \mathrm{kg}$ i.p., twice daily for five days significantly impaired the motor abilities of mice, in terms of decrease in grip strength, development of motor in-coordination and imbalance, decrease in motor activity, and development of immobility assessed using rota rod, beam walking, lateral push and open field tests, respectively. Moreover, 3-nitropropionic acid also led to significant decline in the cognitive ability in mice assessed in terms of decrease in the discrimination index in the object recognition test. In the present study, the object recognition test was used to assess memory instead of maze tests because the evaluation of memory in the maze tests dependent on the presence of the intact motor activity in animals. In the presence of motor impairment, such in this study, the maze tests may not give a true representation of memory. ${ }^{42}$ In contrast, objective recognition test is independent of motor movements; therefore, this test may be more useful to assess memory in animals with motor impairment. ${ }^{42}$ The impairment in motor activities and cognition are the core features of Huntington's disease ${ }^{43}$ and the observed changes in the motor impairment along with decline in memory in response to 3-nitropropionic acid administration in mice may possibly represent the features of HD patients. Amongst the chemical models of HD in animals, 3-nitropropionic acid has been the most widely used to mimic the clinical symptoms of HD in experimental animals. ${ }^{21,22,28}$ Interestingly, the chorea and dance-like features of HD observed in humans are not observed in models of HD in rodents. ${ }^{44}$ Therefore, apart from for the mild trembling, the typical chorealike movements were not observed in this study. Nevertheless, the observed changes in the motor deterioration and cognitive decline in the past study with 3-nitropropionic acid administration have also been reported in other various studies. ${ }^{21,22,28}$

The development of motor impairment in HD patients is secondary to neuronal loss in the basal ganglia, the brain region responsible for fine tuning of the motor movements. ${ }^{2}$ In present study also, administration of 3-nitropropionic acid led to significant neuronal injury and there was a severe loss of neurons in the basal ganglia of the brain assessed in the histopathological studies using hematoxylin and eosin staining along with Nissl staining. The neurons positively stained for hematoxylin and eosin and Nissl were significantly reduced. The hematoxylin and eosin staining is primarily employed to assess the neuronal loss, while Nissl staining is used for the assessment of the extent of atrophy. ${ }^{37,39}$ Moreover, neuron specific enolase (NSE) staining of the brain samples of 3-nitropropionic acid administered animals showed an increase in the number of NSE expressing neurons suggesting the increase in the neuronal injury. NSE has been employed as a reliable marker of neuro-inflammation and neuronal injury. ${ }^{40}$ The caspase- 3 activity was also assessed using immunohistochemistry for the estimation of extent of apoptosis in the brain. Caspases are the proteolytic enzymes which are important role in the digestion of faulty proteins and organelles during the apoptosis process. ${ }^{41}$ The administration of 3-nitropropionic acid resulted in increased number of neurons stained showing caspase-3 activity in comparison with the normal control.

Treatment with 3-methyladenine (15 and $30 \mathrm{mg} / \mathrm{kg}$, i.p.) significantly improved the motor deficits in 3-nitropropionic acid administered mice in terms of improvement in the grip strength, decrease in the immobility, motor activity, motor coordination and balance. The dose-dependent improvement in the motor ability was observed on both $3^{\text {rd }}$ and $6^{\text {th }}$ day of 3-methyladenine injection. 3-Methyladenine treatment also attenuated 3-nitropropionic acidinduced cognitive decline assessed on $6^{\text {th }}$ day in the object discrimination test. Moreover, there was a significant decrease in the neuronal loss, neuronal atrophy, neuro-inflammation and apoptosis in the brain samples of 3-methyadenine treated mice assessed using histopathology and immunohistochemistry. 3-Methyladenine is a widely employed autophagy inhibitor in the experimental studies ${ }^{45,24}$ and it inhibits the initial steps of autophagy by preventing the localization of autophagy proteins to the preautophagosomal membrane. Eventually, it results in the decrease in the formation of autophagosome and thus, autophagy fails to execute its functions. ${ }^{46}$ Accordingly, it may be hypothesized that 3-methyladenine-mediated improvement in the motor and cognitive functions in 3-nitropropionic acid administered animals may be possibly due to inhibition of autophagy. Autophagy is 
a physiological process to remove the faulty/degraded proteins from the cells; ${ }^{7}$ however, excessive activation of autophagy during pathological conditions may also produce deleterious effects due to induction of apoptosis and degradation of essential proteins of the cell. ${ }^{11-13}$ Accordingly, autophagy inhibitors including 3-methyladenine have been reported to produce beneficial effects in different disease states. ${ }^{47,48}$ The role of autophagy in the present study was delineated by assessing the alterations in the levels of a specific marker of autophagy, LC3II. In 3-nitropropionic acid administered mice, there was a significant rise in the levels of LC3II in the brain indicating the activation of autophagy. The administration of 3-nitropropionic acid, a mitochondrial toxin, predisposes the neurons to autophagy. ${ }^{49,50}$

Moreover, the beneficial effects of 3-methyladenine on the motor functions, cognition and neuronal preservation were accompanied by a marked reduction in the levels of autophagy marker, LC3II. Accordingly, it may be proposed that 3-methyadeninemediated inhibition of autophagy may have prevented 3-nitropropionic acid-induced neuronal injury and the development of subsequent deleterious effects on the motor and cognition functions.

To further explore the effect of pharmacological modulation of autophagy in 3-nitropropionic acid model, another widely used autophagy inhibitor, chloroquine, was employed in this study. Chloroquine acts on the later stages of autophagy ${ }^{51,52}$ and inhibits autophagy by inhibiting the fusion of autophagosme and lysosome. ${ }^{53}$ Like 3-methyadenine, treatment with chloroquine phosphate $(50 \mathrm{mg} / \mathrm{kg}$, i.p. $)$ also prevented 3-nitropropionic acid-induced motor and cognitive deterioration on the $3^{\text {rd }}$ and $6^{\text {th }}$ day of treatment. The effects of chloroquine on the neuronal loss, brain atrophy, neuro-inflammation, and apoptosis were consistent with the neuroprotective effects produced by 3-methyladenine treatment in 3-nitropropionic acid administered mice. Nevertheless, the neuroprotective effects of 3-methlyadenine were relatively more prominent than chloroquine. It is interesting to know that the levels of autophagy marker, LC3II depends on the stage of autophagy. Indeed, it is a microtubule associated protein and is closely associated with the autophagosome formation..$^{54}$ Therefore, the autophagy inhibitors which inhibit the early stage of autophagy, such as 3-methladenine decrease the levels of LC3II because of inhibition of autophagosome formation. ${ }^{55}$ On the other hand, the drugs that inhibit autophagy at the later stage i.e. after formation of autophagosome such as chloroquine increases the levels of LC3II. ${ }^{56}$
Accordingly, in the present study the observed rise in the levels of LC3II due to the treatment with chloroquine phosphate may be possibly due to inhibition of autophagy after the formation of autophagosome, which is consistent with earlier reported studies. ${ }^{48}$ To best of our knowledge, it is the first study depicting the beneficial effects of pharmacological inhibitors of autophagy, 3-methyladenine and chloroquine in 3-nitropropionic acid model of HD in mice.

\section{CONCLUSION}

Treatment with 3-methyladenine and chloroquine may improve the symptoms related with Huntington's disease by preventing 3-nitropropionic acid-induced neurodegeneration possibly by inhibiting autophagy.

\section{ACKNOWLEDGEMENT}

The authors are grateful to the Department of Pharmaceutical Sciences and Drug Research, Punjabi University, Patiala, India, for providing the technical facilities for carrying out the research.

\section{CONFLICT OF INTEREST}

The authors declare that there are no conflicts of interest.

\section{ABBREVIATIONS}

H\&E: Hematoxylin and Eosin; LC3II: Light chain 3 kinase; HD: Huntington's disease; CPCSEA: Committee for the Purpose of Control and Supervision of Experiments on Animals; ELISA: Enzyme linked immunosorbent assay; NSE: Neuron specific enolase; ANOVA: Analysis of variance; 3-MA: 3-Methyladenine; CQ: Chloroquine phosphate.

\section{REFERENCES}

1. Bertoglio D, Verhaeghe J, Kosten L, Thomae D, Van der Linden A, Stroobants S, Wityak J, Dominguez C, Mrzljak L, Staelens S. MR-based spatial normalization improves [18F]MNI-659 PET regional quantification and detectability of disease effect in the Q175 mouse model of Huntington's disease. PLOS ONE. 2018;13(10):e0206613. doi: 10.1371/journal. pone.0206613, PMID 30365550.

2. Wright DJ, Renoir T, Gray LJ, Hannan AJ. Huntington's disease: pathogenic mechanisms and therapeutic targets. Adv Neurobiol. 2017;15:93-128. doi: 10.1007/978-3-319-57193-5_4, PMID 28674979.

3. Bondulich MK, Jolinon N, Osborne GF, Smith EJ, Rattray I, Neueder A, Sathasivam K, Ahmed M, Ali N, Benjamin AC, Chang X, Dick JRT, Ellis M, Franklin SA, Goodwin D, Inuabasi L, Lazell H, Lehar A, Richard-Londt A, Rosinski J, Smith DL, Wood T, Tabrizi SJ, Brandner S, Greensmith L, Howland D, Munoz-Sanjuan I, Lee SJ, Bates GP Myostatin inhibition 
prevents skeletal muscle pathophysiology in Huntington's disease mice. Sci Rep. 2017;7(1):14275. doi: 10.1038/s41598-017-14290-3. PMID 29079832.

4. Frank S. Treatment of Huntington's disease. Neurotherapeutics. 2014;11(1):153-60. doi: 10.1007/s13311-013-0244-z, PMID 24366610.

5. Dean M, Sung VW. Review of deutetrabenazine: A novel treatment for chorea associated with Huntington's disease. Drug Des Devel Ther. 2018;12:313-9. doi: 10.2147/DDDT.S138828, PMID 29497277.

6. Coppen EM, Roos RAC. Current pharmacological approaches to reduce chorea in Huntington's disease. Drugs. 2017;77(1):29-46. doi: 10.1007/ s40265-016-0670-4, PMID 27988871.

7. Cai Y, Arikkath J, Yang L, Guo ML, Periyasamy P, Buch S. Interplay of endoplasmic reticulum stress and autophagy in neurodegenerative disorders. Autophagy. 2016;12(2):225-44. doi: 10.1080/15548627.2015.1121360, PMID 26902584.

8. Ravikumar B, Sarkar S, Davies JE, Futter M, Garcia-Arencibia M, GreenThompson ZW, Jimenez-Sanchez M, Korolchuk VI, Lichtenberg M, Luo S, Massey DC, Menzies FM, Moreau K, Narayanan U, Renna M, Siddiqi FH, Underwood BR, Winslow AR, Rubinsztein DC. Regulation of mammalian autophagy in physiology and pathophysiology. Physiol Rev. 2010;90(4):1383435. doi: 10.1152/physrev.00030.2009, PMID 20959619.

9. Shao A, Wang Z, Wu H, Dong X, Li Y, Tu S, Tang J, Zhao M, Zhang J, Hong Y. Enhancement of autophagy by histone deacetylase inhibitor trichostatin $A$ ameliorates neuronal apoptosis after subarachnoid hemorrhage in rats. Mol Neurobiol. 2016;53(1):18-27. doi: 10.1007/s12035-014-8986-0, PMID 25399954.

10. Chen J, Wang L, Wu C, Hu Q, Gu C, Yan F, Li J, Yan W, Chen G. Melatoninenhanced autophagy protects against neural apoptosis via a mitochondrial pathway in early brain injury following a subarachnoid hemorrhage. J Pineal Res. 2014;56(1):12-9. doi: 10.1111/jpi.12086, PMID 24033352.

11. Ye F, Zuo ZY. Anesthetic effects on autophagy. Med Gas Res. 2017;7(3):204-11. doi: 10.4103/2045-9912.215751, PMID 29152214.

12. Orogo AM, Gustafsson ÅB. Therapeutic targeting of autophagy: potential and concerns in treating cardiovascular disease. Circ Res. 2015;116(3):489-503. doi: 10.1161/CIRCRESAHA.116.303791, PMID 25634972.

13. Lee JA, Gao FB. Inhibition of autophagy induction delays neuronal cell loss caused by dysfunctional ESCRT-III in frontotemporal dementia. J Neurosci. 2009;29(26):8506-11. doi: 10.1523/JNEUROSCI.0924-09.2009, PMID 19571141.

14. Zhu H, Tannous P, Johnstone JL, Kong Y, Shelton JM, Richardson JA, Le V, Levine B, Rothermel BA, Hill JA. Cardiac autophagy is a maladaptive response to hemodynamic stress. J Clin Invest. 2007;117(7):1782-93. doi: 10.1172/JCI27523, PMID 17607355.

15. Cui D, Wang L, Qi A, Zhou Q, Zhang X, Jiang W. Propofol Prevents Autophagic Cell Death following oxygen and glucose Deprivation in PC12 Cells and Cerebral ischemia-reperfusion Injury in Rats. PLOS ONE. 2012;7(4):e35324. doi: 10.1371/journal.pone.0035324, PMID 22509406.

16. Cui DR, Wang L, Jiang W, Qi AH, Zhou QH, Zhang XL. Propofol prevents cerebral ischemia-triggered autophagy activation and cell death in the rat hippocampus through the NF-kB/p53 signaling pathway. Neuroscience. 2013;246:117-32. doi: 10.1016/j.neuroscience.2013.04.054, PMID 23644056.

17. Button RW, Luo S, Rubinsztein DC. Autophagic activity in neuronal cell death. Neurosci Bull. 2015;31(4):382-94. doi: 10.1007/s12264-015-1528-y, PMID 26077705.

18. Solesio ME, Saez-Atienzar S, Jordan J, Galindo MF. 3-Nitropropionic acid induces autophagy by forming mitochondrial permeability transition pores rather than activating the mitochondrial fission pathway. $\mathrm{Br} \mathrm{J}$ Pharmacol. 2013;168(1):63-75. doi: 10.1111/j.1476-5381.2012.01994.x, PMID 22509855.

19. González-Polo RA, Bravo-San Pedro JM, Gõmez-Sánchez R, PizarroEstrella E, Niso-Santano M, Fuentes JM. Autophagy, mitochondria and 3-nitropropionic acid joined in the same model. $\mathrm{Br} \mathrm{J}$ Pharmacol. 2013;168(1):60-2. doi: 10.1111/j.1476-5381.2012.02203.x, PMID 22946678.

20. Beal MF, Matson WR, Swartz KJ, Gamache PH, Bird ED. Kynurenine pathway measurements in Huntington's disease striatum: evidence for reduced formation of kynurenic acid. J Neurochem. 1990;55(4):1327-39. doi: 10.1111/j.1471-4159.1990.tb03143.x, PMID 2144582.

21. Török R, Kónya JA, Zádori D, Veres G, Szalárdy L, Vécsei L, Klivényi P. mRNA expression levels of PGC-1a in a transgenic and a toxin model of
Huntington's disease. Cell Mol Neurobiol. 2015;35(2):293-301. doi: 10.1007/ s10571-014-0124-z, PMID 25319408.

22. Veres G, Molnár M, Zádori D, Szentirmai M, Szalárdy L, Török R, Fazekas E, Ilisz I, Vécsei L, Klivényi P. Central nervous system-specific alterations in the tryptophan metabolism in the 3-nitropropionic acid model of Huntington's disease. Pharmacol Biochem Behav. 2015;132:115-24. doi: 10.1016/j. pbb.2015.03.002, PMID 25773340.

23. Wang H, Li Q, Reyes S, Zhang J, Zeng Q, Zhang P, Xie L, Lee PJ, Roncal N, Melendez V, Hickman M, Kozar MP. Nanoparticle formulations of decoquinate increase antimalarial efficacy against liver stage Plasmodium infections in mice. Nanomedicine. 2014;10(1):57-65. doi: 10.1016/j.nano.2013.07.010, PMID 23891618.

24. Duan XC, Wang W, Feng DX, Yin J, Zuo G, Chen DD, Chen ZQ, Li HY, Wang Z, Chen G. Roles of autophagy and endoplasmic reticulum stress in intracerebral hemorrhage-induced secondary brain injury in rats. CNS Neurosci Ther. 2017;23(7):554-66. doi: 10.1111/cns.12703, PMID 28544790.

25. Moulis M, Vindis C. Methods for measuring autophagy in mice. Cells. 2017;6(2):14. doi: 10.3390/cells6020014, PMID 28594368.

26. Vodicka P, Lim J, Williams DT, Kegel KB, Chase K, Park H, Marchionini D, Wilkinson S, Mead T, Birch H, Yates D, Lyons K, Dominguez C, Beconi M, Yue Z, Aronin N, DiFiglia M. Assessment of chloroquine treatment for modulating autophagy flux in brain of WT and HD mice. J Huntingtons Dis. 2014;3(2):159-74. doi: 10.3233/JHD-130081, PMID 25062859.

27. Thomé R, Moraes AS, Bombeiro AL, dos Farias A S, Francelin C, da Costa TA, et al. Chloroquine Treatment Enhances Regulatory $\mathrm{T}$ Cells and Reduces the Severity of Experimental Autoimmune Encephalomyelitis. PLoS One 2013;8(6).

28. Wang L, Wang J, Yang L, Zhou SM, Guan SY, Yang LK, Shi QX, Zhao MG, Yang $Q$, Yang $L$ kun, et al. Effect of Praeruptorin $C$ on 3-nitropropionic acid induced Huntington's disease-like symptoms in mice. Biomed Pharmacother. 2017;86:81-7. doi: 10.1016/j.biopha.2016.11.111, PMID 27939523.

29. Dunham NW, Miya TS. A Note on a Simple Apparatus for Detecting Neurological Deficit in Rats and Mice ${ }^{* *}$ College of Pharmacy, University of Nebraska, Lincoln 8. J Am Pharm Assoc. 1957;46(3):208-9.

30. Luong TN, Carlisle HJ, Southwell A, Patterson PH. Assessment of Motor Balance and Coordination in Mice using the Balance Beam. J Vis Exp 2011;(49).

31. Saklani R, Jaggi A, Singh N. Pharmacological preconditioning by milrinone: Memory preserving and neuroprotective effect in ischemia-reperfusion injury in mice. Arch Pharm Res. 2010;33(7):1049-57.

32. Bederson JB, Pitts LH, Tsuji M, Nishimura MC, Davis RL, Bartkowski H. Rat middle cerebral artery occlusion: evaluation of the model and development of a neurologic examination. Stroke. 1986;17(3):472-6.

33. Menalled L, El-Khodor BF, Patry M, Suárez-Fariñas M, Orenstein SJ, Zahasky B, et al. Systematic behavioral evaluation of Huntington's disease transgenic and knock-in mouse models. Neurobiol Dis. 2009;35(3):319-36.

34. Leger M, Quiedeville A, Bouet V, Haelewyn B, Boulouard M, Schumann-Bard P, et al. Object recognition test in mice. Nat Protoc 2013;8(12):2531-7.

35. Yang Y, Lu F, Zhuang L, Yang S, Kong Y, Tan W, et al. Combined preconditioning with hypoxia and GYKI-52466 protects rats from cerebral ischemic injury by HIF-1a/eNOS pathway. Am J Transl Res. 2017;9(12):5308-19.

36. Klionsky DJ, Abdelmohsen K, Abe A, Abedin MJ, Abeliovich H, Arozena AA, et al. Guidelines for the use and interpretation of assays for monitoring autophagy (3 $3^{\text {rd }}$ edition). Autophagy. 2016;12(1):1-222.

37. Deitch $A D$, Moses MJ. The Nissl substance of living and fixed spinal ganglion cells. II. An ultraviolet absorption study. J Biophys Biochem Cytol. 1957;3(3):449-56.

38. Zille M, Farr TD, Przesdzing I, Müller J, Sommer C, Dirnagl U, et al. Visualizing cell death in experimental focal cerebral ischemia: Promises, problems, and perspectives. J Cereb Blood Flow Metab. 2012;32(2):213-31.

39. Fischer $\mathrm{AH}$, Jacobson KA, Rose J, Zeller R. Hematoxylin and eosin staining of tissue and cell sections. Cold Spring Harb Protoc. 2008;3(5).

40. Haque A, Polcyn R, Matzelle D, Banik NL. New Insights into the Role of Neuron-Specific Enolase in Neuro-Inflammation, Neurodegeneration, and Neuroprotection. Brain Sci 2018;8(2):33.

41. Sanchez Mejia RO, Friedlander RM. Caspases in Huntington's disease. Neuroscientist. 2001;7(6):480-9. 
42. Rosselli FB, Alemi A, Ansuini A, Zoccolan D. Object similarity affects the perceptual strategy underlying invariant visual object recognition in rats. Front Neural Circuits. 2015;9.

43. McColgan P, Tabrizi SJ. Huntington's disease: A clinical review. Eur J Neurol 2018;25(1):24-34.

44. Crook ZR, Housman D. Huntington's Disease: Can Mice Lead the Way to Treatment?. Neuron. 2011;69(3):423-35.

45. Zhang XD, Wang $\mathrm{Y}$, Wang $\mathrm{Y}$, Zhang $\mathrm{X}$, Han $\mathrm{R}, \mathrm{Wu}$ JC, et al. P53 mediates mitochondria dysfunction-triggered autophagy activation and cell death in rat striatum. Autophagy. 2009;5(3):339-50.

46. Pasquier B. Autophagy inhibitors. Cell Mol Life Sci. 2016;73(5):985-1001.

47. Levine B, Kroemer G. Autophagy in the Pathogenesis of Disease. Cell. 2008;132(1):27-42.

48. Lu Q, Harris VA, Kumar S, Mansour HM, Black SM. Autophagy in neonatal hypoxia ischemic brain is associated with oxidative stress. Redox Biol. 2015;6:516-23.

49. Brouillet E, Jacquard C, Bizat N, Blum D. 3-Nitropropionic acid: a mitochondrial toxin to uncover physiopathological mechanisms underlying striatal degeneration in Huntington's disease. J Neurochem. 2005;95(6):1521-40.
50. Filomeni G, De Zio D, Cecconi F. Oxidative stress and autophagy: The clash between damage and metabolic needs. Cell Death Differ. 2015;22(3):377-88.

51. Homewood CA, Warhurst DC, Peters W, Baggaley VC. Lysosomes, pH and the anti-malarial action of chloroquine. Nature. 1972;235(5332):50-2.

52. Helgason GV, Karvela M, Holyoake TL. Kill one bird with two stones: Potential efficacy of BCR-ABL and autophagy inhibition in CML. Blood. 2011;118(8):2035-43.

53. Galluzzi L, Pedro BSJM, Levine B, Green DR, Kroemer G. Pharmacological modulation of autophagy: therapeutic potential and persisting obstacles. Nat Rev Drug Discov. 2017;16(7):487-511.

54. Rubinsztein DC, Shpilka T, Elazar Z. Mechanisms of autophagosome biogenesis. Curr Biol. 2012;22(1):R29-34.

55. Ding D, Xu S, Zhang H, Zhao W, Zhang X, Jiang Y, et al. 3-methyladenine and dexmedetomidine reverse lipopolysaccharide-induced acute lung injury through the inhibition of inflammation and autophagy. Exp Ther Med. 2018;15(4):3516-22.

56. Kimura T, Takabatake Y, Takahashi A, Isaka Y. Chloroquine in cancer therapy: A double-edged sword of autophagy. Cancer Res. 2013;73(1):3-7.

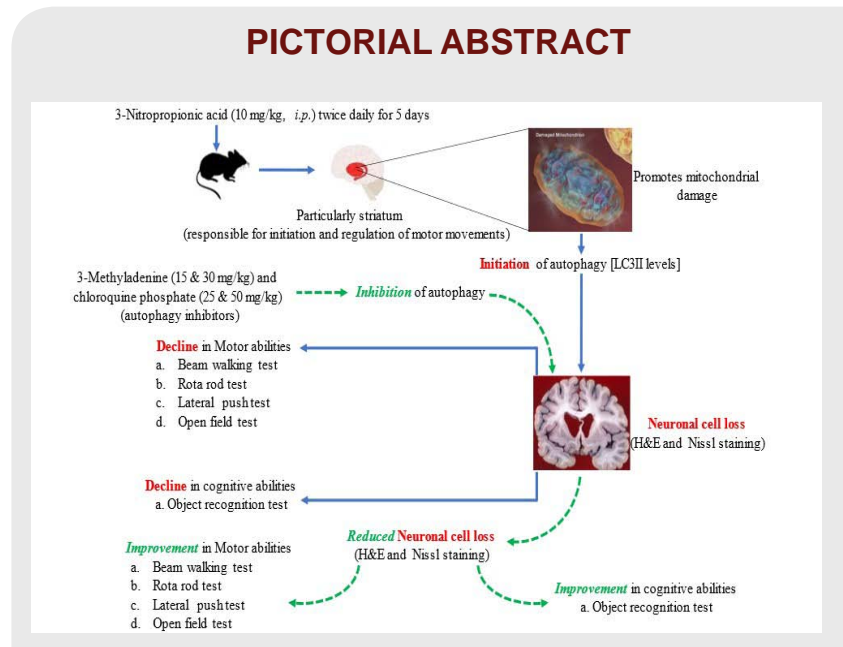

\section{SUMMARY}

In summary, the present study showed that administration of autophagy inhibitors, 3-methyladenine and chloroquine phosphate significantly inhibited the process of autophagy in mice with 3-nitropropionic acid induced Huntington's disease. This resulted in reduced neuronal loss ultimately improving the behavioral parameters (beam walking, rota rod, lateral push and open field test) and cognitive abilities (object recognition test) of the animals. Therefore, inhibition of autophagy may slow down progression of the disease.

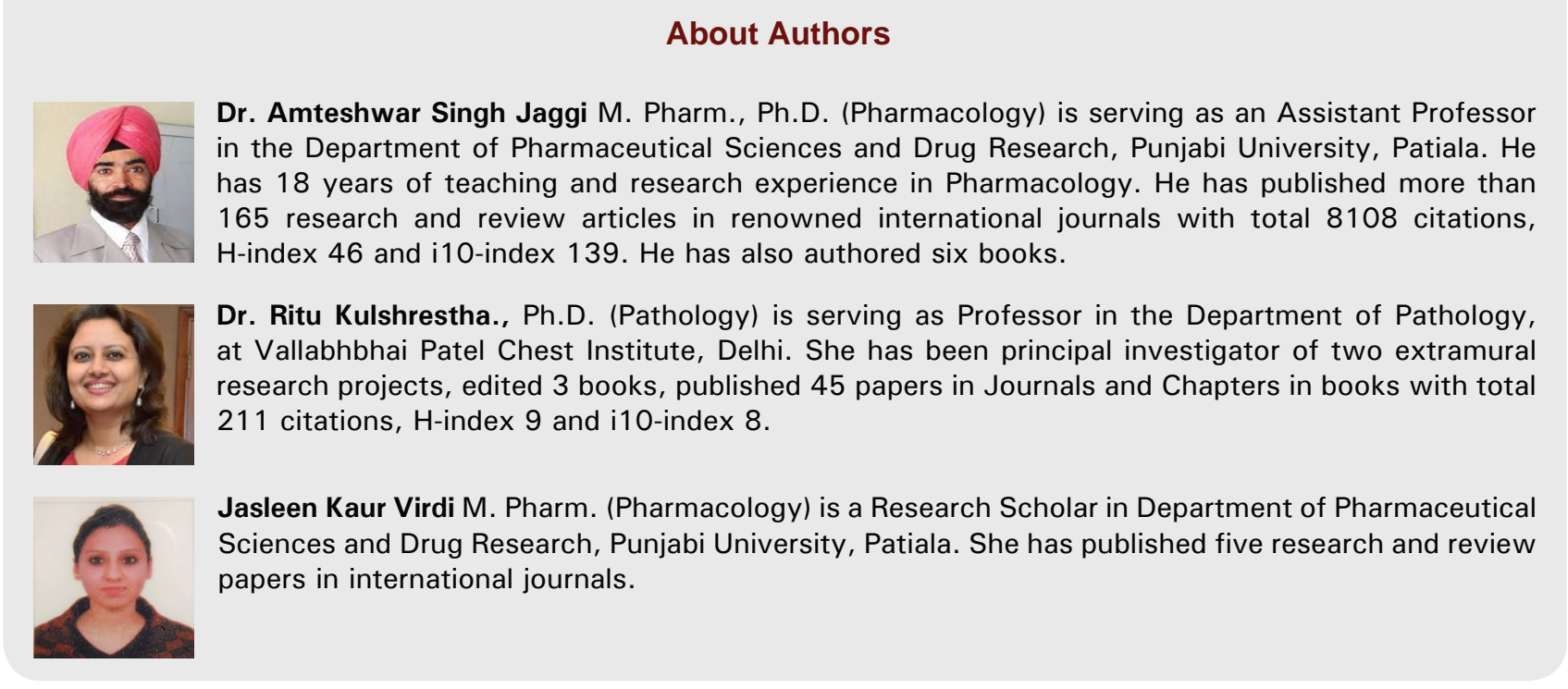

Cite this article: Virdi JK, Kulshrestha R, Jaggi AS. Ameliorative Effects of 3-methyladenine and Chloroquine in 3-nitropropionic-induced Huntington's Disease like Symptoms in Mice. Indian J of Pharmaceutical Education and Research. 2021;55(4):1037-47. 